\title{
Aproximaciones al estudio del pódcast como documento sonoro de origen digital
}

\author{
Perla Olivia Rodríguez Reséndiz*
}

Artículo recibido:

24 de agosto de 2021

Artículo aceptado:

28 de octubre de 2021

Artículo de investigación

\section{Resumen}

La era dorada del pódcast sonoro es la forma como se califica a la segunda década del siglo XXI. Este periodo se caracteriza por la proliferación, diversidad y amplia distribución de este tipo de materiales sonoros, que son evidencia de la creación, pensamiento y expresión artística de la sociedad. El pódcast, además de ser un medio a través del cual se transmite información audible, es un tipo de documento de origen digital cuya naturaleza documental ha sido poco estudiada. Este artículo tiene como objetivo determinar algunas de las características del pódcast como documento sonoro de origen digital y con ello contribuir a establecer las bases para su preservación. Para ello, se presenta el

* Instituto de Investigaciones Bibliotecológicas y de la Información, Universidad Nacional Autónoma de México, México perla@iibi.unam.mx 
marco conceptual, el origen y evolución del pódcast, se define la estructura y el soporte del pódcast como objeto digital, se alude a su relevancia como medio que aborda contenidos de actualidad e interés social, y se señala el riesgo de pérdida de este tipo documental.

Palabras clave: Pódcast Sonoro; Archivo Sonoro; Preservación; Preservación Digital Sonora

\title{
Approaches to the study of podcast as a born digital sound document \\ Perla Olivia Rodríguez Reséndiz
}

\begin{abstract}
The golden age of the sound podcast is how the second decade of the 21st century is described. This period is characterized by the proliferation, diversity and wide distribution of this kind of sound materials that are evidence of the creation, thinking and artistic expression of society. The podcast besides being a medium through which audible information is transmitted, is a kind of born digital document whose documentary nature has been studied very little. The purpose of this article is to determine some of the characteristics of podcast as a born digital sound document, and defines the structure and the medium of podcast as a digital object, referring to it as a medium that deals with current affairs and social interest and points out the risk of loss of this type of documentary.
\end{abstract}

Keywords: Podcast; Sound Archive; Preservation; Sound Digital Preservation

\section{INTRODUCCIÓN}

$\mathrm{E}^{1}$ 1 documento sonoro es el medio a través del cual se transmite información audible como evidencia de la creación, pensamiento y expresión artística de la sociedad. Se define como el registro de información sonora creado con un propósito intelectual determinado que adquiere valor documental cuando se incorpora en un archivo sonoro y reúne cuatro cualidades: contenido, estructura, contexto, y estar fijado en uno o más soportes (analógico o 
digital). El contenido es la información sonora registrada relativa a uno o más temas. La estructura se refiere a la forma de la grabación a partir del uso de los elementos del lenguaje sonoro (voz, música, silencios y efectos), así como el género y el formato en que ha sido producido el documento; esta cualidad define las características físicas e intelectuales del documento como evidencia (Miller, 2017). El contexto es el conjunto de datos externos de las circunstancias en las cuales fue grabado el documento, es la información relativa al cómo, dónde, cuándo, qué, quién y por qué se creó el registro. Son los metadatos a partir de los cuales es posible identificar y recuperar el documento. La información de contexto es una de las cualidades más significativas de todo documento sonoro (Rodríguez, 2020).

El soporte es el artefacto en que fue registrado el sonido. Se distinguen dos épocas en la producción de soportes: analógicos, producidos desde finales del siglo XIX hasta los últimos años del XX, y digitales, que caracterizan al siglo XXI. Estos últimos se agrupan en discos ópticos (CD y DVD) y de origen digital, también denominados nativos digitales (Edmondson, 2018).

Si en el siglo pasado la conservación de los soportes analógicos (cilindros, discos, cintas magnetofónicas, entre otros) fue la tarea documental prioritaria porque la noción de patrimonio consideraba tanto el soporte físico como el contenido grabado, a partir de la digitalización y con la irrupción de los nativos digitales la impronta de la preservación es garantizar la permanencia de los contenidos, porque a diferencia del documento analógico, el de origen digital no tiene un soporte único y definitivo. Se preserva gracias a la migración sistemática y se conserva la autenticidad e integridad del documento independientemente del soporte en que se copie, siempre y cuando no se altere o modifique el contenido.

Los documentos de origen digital son objetos digitales intangibles que se crean en una amplia gama de géneros y formatos. Entre otros, se producen programas de radio, grabaciones de campo, audiolibros, grabaciones de audio social y pódcast. Este último destaca como recurso contemporáneo de información sonora, si bien conviene señalar que también se producen pódcast en video. Este artículo se centra en las producciones sonoras.

En torno al pódcast sonoro existe una amplia gama de investigaciones. Se ha estudiado su origen y evolución (Berry, 2016; Gallego, 2010; Orrantia, 2019; Rowell, 2020). Se ha escrito en relación al crecimiento exponencial de la audiencia, de las producciones, así como de las plataformas a través de las cuales se distribuye (Locker, 2018; Morris, Hansen y Hoyt, 2019). Ha sido analizado a partir de su vínculo con el medio radiofónico y como un elemento clave para comprender los nuevos medios digitales (Martínez-Costa, Müller y Villar, 2019; Salaverría, Martínez-Costa y Breiner, 2018), así como la 
amplia gama de posibilidades de uso educativo, comercial (Musson, 2019) y político. También se han determinado sus características como un tipo de producción que expresa la transición del lenguaje sonoro al transmedia (García-Marín y Aparici, 2018; Steven y Johnson, 2010). Abundan los estudios de caso en relación con la producción y el consumo de pódcast en Estados Unidos y en Europa, en particular en España.

Desde el inicio de su expansión, atrajo la atención de bibliotecarios y archivistas. Cordes (2008) expuso sus posibilidades como un recurso de información en las bibliotecas por el bajo costo, así como por las posibilidades tecnológicas para la distribución de contenidos. Fue valorado como sustituto del cassette, soporte de apoyo para que los estudiantes escucharan las clases a las cuales no podían asistir (Read, 2021). También se formularon los primeros problemas asociados con el almacenamiento digital, el archivado, los metadatos, la creación de catálogos y la indización (Gordon-Murnane, 2005). Sin embargo, se carece de investigaciones en torno a la naturaleza documental del pódcast como documento de origen digital y a su preservación digital.

\section{Metodología}

La abundancia de pódcast es un fenómeno reciente que ha atraído el interés científico, aun cuando resulta complejo debido a que es un objeto de estudio en proceso de evolución. Derivado de esta condición y con el objetivo de determinar algunas de las características del pódcast como documento sonoro de origen digital y, con ello, contribuir a establecer las bases para su preservación, fue necesario recurrir a la investigación cualitativa. Se analizó bibliografía y documentación publicadas a partir de 2005, debido a que los primeros pódcast se difundieron en internet a partir de 2004. A partir de las referencias consultadas se construyó el marco conceptual, se estableció el origen y evolución del pódcast. Después, a fin de comprender las características del pódcast como documento sonoro de origen digital, se utilizaron las propiedades que Manovich (2005) estableció en relación con los objetos digitales.

Además, se empleó la escucha atenta como técnica de análisis de contenido de los documentos sonoros en relación con los temas tratados, la estructura narrativa y la calidad de la producción. Se identificaron y escucharon materiales documentales y de ficción de ocho series que comprenden 54 producciones. Se eligieron producciones en español, con alta calidad de producción y cuyos temas son de relevancia e interés social. Las observaciones derivadas de este proceso hicieron posible avisorar la relevancia del pódcast como un documento de origen digital que recupera contenidos de interés y relevancia social contemporánea. 


\section{CONTEXTO y DESARROLLO DEL PÓDCAST}

El pódcast sonoro comenzó a tener presencia en internet en 2004. "Audioblogging? Podcasting? GuerrillaMedia?”, preguntó el periodista británico Ben Hammersley en el artículo "Audible revolution", al cuestionar cómo nombrar al tipo de producción sonora que transmite "la intimidad de la voz, la interactividad de un weblog y la conveniencia y portabilidad de una descarga de MP3" (Hammersley, 2004. Traducción propia). Este autor acuñó la noción de pódcast en su artículo y un año después el New Oxford American Dictionary incorporó el término. Desde su origen, este tipo de producción fue disruptiva con las formas de comunicación de los medios tradicionales y cimentó las bases para la revolución audible del siglo XXI.

A partir de 2008, los pódcast comienzan a poblar diversas plataformas y a partir de 2015 se observa un crecimiento sostenido (Listen notes, 2021). En 2020, en el marco de la pandemia por COVID-19, se incrementó la cantidad de materiales en 300 \% en relación con el año anterior. En el primer semestre de 2021 se cuantificaron 2621086 pódcast y 111578161 episodios (Listen notes, 2021). Spotify documenta la existencia de 70 millones de tracks, de los cuales 2.9 millones son pódcast (Spotify, 2021). Los principales idiomas de los pódcast son inglés, español y portugués. La abundancia y diversidad de temas de este tipo de materiales sonoros han merecido que la era actual sea nominada como la era dorada del pódcast y de la audiofonía.

La palabra pódcast es un anglicismo que no tiene un equivalente en español. Se usa para nombrar a un archivo de sonido portable, descargable, al que se pueden suscribir los usuarios (Gallego, 2010; Sellas y Solà, 2019; Orrantia, 2019), y como un canal para ofrecer a los oyentes lo que demandan (Blanco et al., 2013). Este "archivo sonoro, grabado — en algunos casos con diseño-, se distribuye por sus autores a través de internet con una periodicidad concreta (diaria, semanal, mensual...)" (Orrantia, 2019: 102).

El pódcast es el archivo en formato MP3, o bien, el paquete de información y metadatos que se almacena y distribuye a través de plataformas web para su descarga y escucha en dispositivos tecnológicos (teléfonos móviles, tablets, computadoras, entre otras).

\section{LA ESTRUCTURA Y EL SOPORTE DEL PÓDCAST COMO OBJETO DIGITAL}

Los documentos sonoros de origen digital carecen de las restricciones físicas de los soportes analógicos (Wiencek, 2018) y su preservación se basa en los principios que garanticen la permanencia de este tipo de materiales (Rodríguez, 
2017). Son definidos como paquetes de información (ISO, 2012) que se preservan durante el ciclo de vida (Bradley et al., 2011). Además, son objetos digitales que se caracterizan por su representación numérica, modularidad, automatización, variabilidad y transcodificación (Manovich, 2005).

El pódcast es un nuevo tipo de documento sonoro de origen digital cuya estructura puede ser analizada a partir de sus propiedades como objeto digital. La información se representa de forma numérica en bits mediante un código binario, es decir, unos (1) y ceros (0). Es fractual o modular dado que se produce en un determinado formato digital y su resolución se determina a través de la frecuencia de muestreo y el número de bits por muestra. Se puede fragmentar y cada parte combinarse a su vez con otros elementos digitales sonoros o bien con otros lenguajes, como los hipertextuales, gráficos o de imagen en movimiento, por ejemplo. Las tecnologías de edición sonora favorecen que se pueda copiar, pegar, cortar e incluso transformar.

De hecho, "la narrativa actual del pódcasting desborda el carácter exclusivamente sonoro de sus inicios al construir productos mediáticos y culturales multilenguaje. Si bien el objeto nuclear de estos trabajos continúa siendo el contenido sonoro, los pódcasters amueblan su universo narrativo con producciones que utilizan otros formatos mediáticos. El uso de fotografías, texto y recursos audiovisuales contribuyen a extender, comprimir o repetir los contenidos que provee este medio" (García-Marín y Aparici, 2018: 1080). La narrativa transmedia "es una estructura narrativa particular que se expande a través de diferentes lenguajes (verbal, icónico, etc.) y medios (cine, cómic, televisión, videojuegos, etc.) [...] no es sólo la adaptación de un medio a otro [...]. Esta dispersión textual es una de las fuentes de complejidad más importantes de la cultura popular contemporánea" (Scolari, 2009: 587. Traducción propia). La inserción del lenguaje transmedia modifica la noción de documento sonoro y evidencia el tránsito al tratamiento documental hipermedios.

La representación numérica y la modularidad del pódcast favorecen la automatización de operaciones relacionadas con la creación, manipulación y acceso a la información digital. Uno de los rasgos distintivos del pódcast ha sido su mecanismo de distribución. Surgió como tecnología, antes de la llegada de las redes sociales y de la nube como dispositivo de almacenamiento. La distribución automática de archivos de audio añadidos a un RSS (Real Simple Syndication) mediante la etiqueta <enclosure> posibilitó la descarga de noticias en audio, su inserción y escucha en un reproductor portátil; así se dio origen al pódcast (Gallego, 2010) y al mismo tiempo se erigió como una arquitectura técnica descentralizada, gracias a la cual los contenidos de audio se almacenan en toda la web y se enlazan entre sí a través de RSS (Sullivan, 2019). La fragmentación en la distribución y acceso desafió en un inicio la 
atracción centrípeta y la tendencia a la creación de plataformas concentradoras de contenidos digitales (Sullivan, 2019). No obstante, a partir de 2005, se advierte un cambio derivado de la proliferación de plataformas de pódcast. La automatización también se vincula con la incorporación de tecnologías para la identificación y reconocimiento de voz a través de técnicas de inteligencia artificial, por ejemplo.

La variabilidad es otra cualidad del pódcast sonoro, lo que significa que un documento sonoro nativo digital puede existir en diferentes formatos, es decir, en paquetes de información almacenados como archivos de datos o enviados como flujos de información a través de redes (también conocidos como bitstreams, byte streams) (Library of Congress, 2021). Los formatos pueden crearse sin reducción de datos o bien mediante técnicas de codificación perceptual (lossy codecs); es decir, reducción o pérdida de datos. Los formatos con reducción de datos tienen pérdida de información original (Bradley et al., 2011) y se les llama comprimidos. Además, pueden ser propietarios o abiertos. En el primer caso es posible que en un futuro se solicite algún tipo de pago para la reproducción de los contenidos, en tanto que los abiertos ofrecen de forma libre y gratuita toda la información sobre el formato (Rodríguez, 2017).

Las directrices para transferir contenidos grabados en soportes analógicos por medio de la digitalización recomiendan el uso de formatos abiertos y sin compresión, como son el WAVE y el AIFF, en resolución de cuando menos $48 \mathrm{kHz}$ y 24 bits (Bradley et al., 2011). Este principio ha guiado la preservación de documentos de origen digital. Sin embargo, contrario a lo recomendado, la producción y distribución de pódcast se basa en formatos de compresión como el MP3. El uso generalizado de este formato es resultado de la posibilidad de transcodificación, es decir, que el objeto digital se grabe en un formato sin compresión como puede ser el WAVE o AIFF y se convierta en otro, como el MP3, que facilita su portabilidad, reproducción y uso. El éxito y desarrollo del pódcast sonoro se basa en esta posibilidad.

El uso del MP3 confronta los principios de integridad, autenticidad y origen de procedencia que guían el trabajo en el archivo. Ante el riesgo de pérdida de este tipo de documentos, en la práctica se recomienda que si los documentos fueron creados en formatos de compresión deben ser preservados, siempre y cuando la pérdida sea aceptada por el autor, el editor y el usuario principal (Library of Congress, 2021). Esta podría ser una excepción en caso de no contar con los formatos recomendados para la preservación (WAVE y AIFF). 
La temática de los pódcast es diversa (sociedad y cultura, educación, arte, negocios, religión y espiritualidad, comedia, salud y bienestar, noticias, música, ocio, deportes, televisión y cine, niños, familia y otros). Destacan por su volumen las producciones con temas de sociedad y cultura; se identifican 433106 pódcast (Listen notes, 2021) en dos géneros: documentales y ficciones sonoras.

Muchas de estas producciones se distinguen por la originalidad y la calidad sonora. Los documentales se sustentan en una amplia investigación periodística e incorporan como parte de su narrativa testimonios y materiales de archivo. Los enfoques son originales y únicos. Las producciones son realizadas por profesionales o por aficionados. Pueden basarse o no en guiones, carecen de una duración determinada y el escucha puede suscribirse para recibir el contenido actualizado (Orrantia, 2019).

Son ejemplo de pódcast documentales que abordan temas de interés social contemporáneo "Cruces en el desierto", que narra la crisis humanitaria que se vive en la frontera de Estados Unidos y México (Maxwell, 2021a). "Una familia al centro de la okupa" refiere la ocupación feminista de la Comisión Nacional de Derechos Humanos en la Ciudad de México (Knoll, 2021). "59 balas" narra la historia de Alex Nieto, un joven latino de 28 años a quien la policía de San Francisco mató por considerarlo sospechoso (Maxwell, 2021b). "La lista", serie que da cuenta de la vida de un subteniente del ejército mexicano acusado por colaborar con narcotraficantes (Así como suena y El País, 2021). "La teoría del paréntesis" es la historia de los adultos mayores que quedaron atrapados en las residencias españolas durante la pandemia por COVID-19 (Quesada y Peinado, 2021). Los pódcast también son medios de denuncia social, como lo demuestra la serie "Colombia protesta", a través de la cual se ofrecen testimonios y voces en torno a las movilizaciones en Colombia (Godínez, 2019).

Los pódcast de ficción han atraído la atención de los escuchas como un alternativa de entretenimiento. En esta categoría puede citarse "El estallido de las cosas", serie de pódcast de ficción en la que los objetos son protagonistas durante el estallido social ocurrido en Chile de 2019. Destaca la alta calidad en la producción sonora y el diseño de la página web con animaciones en 3D e hipertextos (Court y Piritz, 2021). Otro ejemplo es "El gran apagón", contada en tres temporadas que reconstruyen los efectos de una tormenta solar que deja al planeta en completa oscuridad, sin internet, telefonía, televisión ni luz eléctrica (Alonso, 2017). 
Además de proporcionar acceso a la producción sonora, en las páginas de estas series se publican fotografías, hipertextos y en algunos casos animaciones en 3D. El pódcast es un documento sonoro de origen digital con información complementaria transmedia, cuyo tratamiento documental debe considerar también tales contenidos. Esta característica afecta la forma de la preservación del documento de origen digital porque además del sonido es necesario acopiar y preservar los elementos gráficos, de imagen en movimiento e hipertextuales que forman parte de la producción.

Las producciones antes citadas son una muestra de la importancia que tiene el pódcast como documento que recupera temas de interés social que no se transmiten en los circuitos tradicionales de difusión de información sonora y de acceso a documentos audibles.

\section{UBICUIDAD Y RIESGO DE PÉRDIDA}

El éxito y la popularidad de un pódcast no significa que sea preservado. Son tipos documentales frágiles y nadie podría garantizar que las producciones actuales sean escuchadas de nuevo en un año. Las razones son diversas. Los programas informáticos (software) para crearlos, editarlos y reproducirlos pueden salir del mercado y dejar de funcionar. Las plataformas de distribución pueden interrumpirse debido a la falta de solvencia económica. Los sitios web pueden cerrar o ser vendidos a una empresa más grande. Los datos para la recuperación pueden no ser reconocibles si carecen de formatos interoperables. Los sistemas de almacenamiento digital pueden colapsar y dejar de operar. De un momento a otro, puede suceder un fallo de energía, un error humano, problemas técnicos, dificultades económicas o incluso que los formatos digitales cambien. Las personas que publican pódcast sonoros pueden eliminar las grabaciones en cualquier momento. Incluso puede ser que se carezca de los derechos de autor para continuar con la publicación (Bamberger y Brylawski, 2010).

La libertad de distribución y la facilidad con que se crean los pódcast probablemente inciden en que muchos podcasteros omitan preservarlos. Se desconoce que muchas de las producciones son únicas, poseen valor documental y constituyen un estilo de producción sonora característica del siglo XXI. Son formatos emergentes que los investigadores, estudiosos de los medios de comunicación, así como los humanistas digitales, entre otros, estudiarán y referenciarán. 
Se producen un vasto volumen de pódcast, se distribuyen en una amplia gama de plataformas y desaparecen de un momento a otro. Un ejemplo de ello es Daily Source Code, una de las primeras series con más de 860 episodios que produjo Adam Curry, entre 2004 y 2013. Curry publicó en 2014 un mensaje en su sitio web y en Twitter en el que preguntó a sus más de 40000 seguidores si alguien tenía el archivo completo de la serie. "El programa de Curry se cita a menudo como uno de los primeros pódcast populares de la historia y, sin embargo, incluso Curry no sabía dónde estaban estos archivos históricamente significativos" (Morris, Hansen y Hoyt, 2019: 12).

En una situación similar se encuentran los productores de pódcast independientes quienes carecen de medios e infraestructura para identificar, almacenar y preservar de forma adecuada sus audios. En la búsqueda de alternativas de solución se creó Preserve this Podcast, proyecto financiado por la Fundación Andrew W. Mellon y auspiciado por el Consejo de Bibliotecas Metropolitanas de Nueva York (METRO), con el fin de ayudar a los podcasteros a proteger su trabajo contra las amenazas del deterioro digital. El proyecto comenzó en enero de 2018 y concluyó en 2020 (METRO, 2021). En la página del proyecto se ofrecen recomendaciones básicas para que los podcasteros apliquen prácticas de preservación (METRO, 2021). El riesgo de pérdida de los pódcast es significativo. Son contadas las iniciativas de preservación en repositorios de acceso abierto como Internet Archive que, hasta abril de 2021, reportó la existencia de 3874936 ítems (Internet Archive, 2021). Muchas grabaciones se han perdido.

\section{Conclusiones}

El pódcast sonoro es un nuevo tipo de documento nativo digital cuyo origen y desarrollo se sitúa en el ecosistema digital del siglo XXI. Su estructura puede ser estudiada a partir de sus propiedades como objeto digital (representación numérica, modularidad, automatización, variabilidad y transcodificación) y de acuerdo con los contenidos tratados. Al igual que otros documentos nativos digitales, posee propiedades de representación binaria. $\mathrm{Su}$ esencia son los bits y se representan como unos (1) y ceros (0). Es fractual, condición que determina su formato y resolución. Puede ser combinado con otros elementos sonoros o bien crear una narrativa transmedia, acompañarse de otro tipo de información, como puede ser textual, gráfica o hipertexutal, condición que favorece su publicación en un entorno transmedia e implica un tratamiento documental hipertextual. 
Su carácter de representación binaria y fractual favorece la automatización, y con ello, la distribución, búsqueda y recuperación como información digital. La variabilidad y transcodificación del pódcast sonoro como objeto digital le confieren la posibilidad de que pueda ser convertido en diferentes formatos. De estos prolifera el MP3, formato de compresión que facilita la distribución y ensancha el acceso, aun cuando contraviene las recomendaciones de preservación digital en formatos abiertos y sin compresión.

El pódcast es un tipo de documento sonoro de origen digital con valor social y cultural. Se producen pódcast en un amplio rango de temas de interés contemporáneo. En este tipo de materiales se registran aspectos poco abordados por otros medios. Por lo tanto, es un testimonio, constituye una forma de patrimonio digital y un bien cultural en alto riesgo de pérdida debido a que su presencia es disruptiva y su preservación está fuera de los principios de la preservación digital. La desaparición de los pódcast sonoros podría significar la desaparición de una forma del patrimonio digital contemporáneo.

Se estima que su permanencia sea similar a la de los cilindros de cera, es decir, los incunables sonoros. Esto significa que en el próximo siglo, en caso de que no se desarrollen principios para su preservación, sólo se preservará un porcentaje bajo de este tipo documental. Una de las primeras acciones para intentar revertir la tendencia de riesgo de pérdida es reconocer las cualidades documentales del pódcast, lo que conllevará a un cambio en los métodos habituales de preservación digital sonora.

\section{REFERENCIAS}

Alonso, Ana, dir. 2017. “El gran apagón”, en Podium Podcast, tres temporadas. Fecha de consulta: 15 de enero de 2021. https://www.podiumpodcast.com/el-gran-apagon/

Así como suena y El País, prod. 2021. "La lista”, en Así como suena, nueve episodios. Fecha de consulta: 28 de junio de 2021. https://lalista.asicomosuena.mx

Bamberger, Rob y Sam Brylawski, eds. 2010. The state of recorded sound preservation in the United States: A national legacy at risk in the digital age. Council on Library and Information Resources: Library of Congress. Fecha de consulta: 13 de agosto de 2021.

https://www.clir.org/pubs/reports/pub148/

Berry, Richard. 2016. "Podcasting: Considering the evolution of the medium and its association with the word radio". The Radio Journal: International Studies in Broadcast and Audio Media 14 (1): 7-22. https://doi.org/10.1386/rjao.14.1.7_1 
Blanco, María, Alfonso López, Emma Rodero y Luis Corredera. 2013. "Evolución del conocimiento y consumo de podcasts en España e Iberoamérica”. Trípodos 33, 53-72.

http://www.tripodos.com/index.php/Facultat_Comunicacio_Blanquerna/article/view/97

Bradley, Kevin, Mike Casey, Stefano Cavaglieri, Chris Clark, Dietrich Schüller, Matthew Davies, Jouni Frilander, Lars Gaustad, Ian Gilmour, Albrecht Häfner, Nadia Wallaszkovits et al. 2011. Directrices para la producción y preservación de objetos digitales de audio. South Africa: IASA.

Cordes, Sean. 2008. "Podcast Pilots for Distance Planning, Programming, and Development”. Journal of Library E Information Services In Distance Learning 2 (4): 79-91. https://doi.org/10.1300/J192v02n04_07

Court, María y Trinidad Piritz, dir. 2021. "El estallido de las cosas + breves anotaciones de escucha”, en Border, siete episodios. Fecha de consulta: 28 de junio de 2021. https://us18.campaign-archive.com/?u=a1fc6541bdf3ffa7049c10903\&id=107e400220

Edmondson, Ray. 2018. Filosofía y principios de los archivos audiovisuales, 3a. ed. México: Unesco.

https://unesdoc.unesco.org/ark:/48223/pf0000243973

Gallego, Ignacio. 2010. Podcasting: Nuevos modelos de distribución y negocio para los contenidos sonoros. España: UOC.

https:/www-digitaliapublishing-com.pbidi.unam.mx:2443/visorreadspeaker/20479

García-Marín, David y Roberto Aparici. 2018. "Nueva comunicación sonora. Cartografía, gramática y narrativa transmedia del podcasting". El profesional de la información 27 (5): 1071-1081. https://doi.org/10.3145/epi.2018.sep.11

Godínez, Francisco, prod. y dir. 2019. "Colombia-protesta”, en CPR, ocho episodios. Centro de Producciones Radiofónicas. Fecha de consulta: 12 de julio de 2021. https://cpr.org.ar/tag/colombia-protesta/

Gordon-Murnane, Laura. 2005. "Saying I do to podcasting: Another next big thing for librarians?" Searcher the Magazine for Database Professionals 13 (6): 44-51.

Hammersley, Ben. 2004. "Why online radio is booming". The Guardian. http://www.theguardian.com/media/2004/feb/12/broadcasting.digitalmedia

Internet Archive. 2021. "Pódcast". Fecha de consulta: 15 de mayo de 2021. https://archive.org/search.php?query=podcast

ISO (International Organization for Standarization). 2012. Space Data and Information Transfer Systems -Open Archival Information System (OAIS)-Reference Model, ISO 14721:2012, París: ISO.

Knoll, Andalusia, prod. 2021. "Una familia al centro de la okupa”, en Las raras pódcast, temporada V. Fecha de consulta: 5 de julio de 2021. https://lasraraspodcast.com/episodio/una-familia-al-centro-de-la-okupa/

Library of Congress. 2021. Sustainability of Digital Formats: Planning for Library of Congress Collections. Fecha de consulta: 3 de mayo de 2021.

https://www.loc.gov/preservation/digital/formats/

Listen notes. 2021. "Podcast Stats: How many podcasts are there?". Fecha de consulta: 20 junio de 2021. https://www.listennotes.com/podcast-stats/ 
Locker, Melissa. 2018. “Apple's podcasts just topped 50 billion all-time downloads and streams". Fast Company. Fecha de consulta: 28 de junio de 2021. https://www.fastcompany.com/40563318/apples-podcasts-just-topped-50-billion-all-time-downloads-and-streams

Manovich, Lev. 2005. El lenguaje de los nuevos medios de comunicación: La imagen en la era digital. Buenos Aires: Paidós.

Martínez-Costa, María del Pilar, María José Müller y Martha Villar. 2019. "La expansión del podcast en la radio pública: estudio comparado de Radio Nacional Argentina y Radio Nacional de España”. Hipertext.net (20): 55-67. https://doi.org/10.31009/hipertext.net.2020.i20.05

Maxwell, Dennis, prod. 2021a. "Cruces en el desierto", en Las raras podcast, temporada IV. Fecha de consulta: 28 de mayo de 2021. https://lasraraspodcast.com/episodio/cruces-en-el-desierto/

Maxwell, Dennis, prod. 2021b. "59 balas”, en Las raras podcast, temporada V. Fecha de consulta: 28 de junio de 2021. https://lasraraspodcast.com/episodio/59-balas/

METRO (Consejo de Bibliotecas Metropolitanas de Nueva York). 2021. Preserve This Podcast. Fecha de consulta: 1 de junio de 2021. http://preservethispodcast.org/

Miller, Laura. 2017. Archives: Principles and Practices, 2a. ed. Cambridge: Facet Publishing. Morris, Jeremy Wade, Samuel Hansen y Eric Hoyt. 2019. "The PodcastRE Project: Curating and Preserving Podcasts (and Their Data)". Journal of Radio and Audio Media 26 (1): 8-20. https://doi.org/10.1080/19376529.2019.1559550

Musson, Dave. 2019. "Expertise in Your Ears; Why You Should Jump on the Podcasting Bandwagon", en Social Media in Higer Education. Case studies, reflections and analysis, editado por Chris Rowell, 199-210. Cambridge, UK: Open Book Publishers.

Orrantia, Andoni. 2019. Diez claves para contar buenas historias en podcast. O cómo producir contenidos en un entorno digital cambiante. España: Editorial UOC.

Quesada, Juan Diego y Fernando Peinado, guion y narrad. 2021. "Podcast: La teoría del paréntesis I Covid-19 en las residencias de ancianos", en El País y Podium Podcast, tres capítulos. Fecha de consulta: 17 de mayo de 2021. https://elpais.com/especiales/2021/covid-19-en-las-residencias-de-ancianos/

Read, Brock. 2021. "Abandoning Cassette Tapes, Purdue U. Will Podcast Lectures in Almost 50 Courses". Chronicle of Higher Education 52 (3): A32. https://search-ebscohost-com.pbidi.unam. $\mathrm{mx}$ :2443/login.aspx?direct=true \&d$\mathrm{b}=$ edselc\&AN=edselc.2-52.0-33751162131\&lang=es \&site=eds-live

Rodríguez, Perla. 2017. "Propuesta de principios que se deben tener en cuenta para la preservación de documentos sonoros de origen digital". Anales de Documentación 20 (2). https://doi.org/10.6018/analesdoc.20.2.272181

Rodríguez, Perla. 2020. El archivo digital sonoro. México: UNAM. https://doi.org/10.22201/iibi.9786073041430e.2020

Rowell, Chris. 2020. "From a Tweet to a Blog to a Podcast". Social Media in Higher Education: Case Studies, Reflections and Analysis, 3-13. Cambridge: Open Book Publishers. http://books.openedition.org/obp/8521 
Salaverría, Ramón, María del Pilar Martínez-Costa y James Breiner. 2018. "Mapa de los cibermedios de España en 2018: Análisis cuantitativo”. Revista Latina de Comunicación Social 73: 1034-1053. https://doi.org/10.4185/RLCS-2018-1295

Scolari, Carlos. 2009. "Transmedia Storytelling: Implicit Consumers, Narrative Worlds, and Branding in Contemporary Media Production”. International Journal of Communication 3, 586-606. Fecha de consulta: 30 de marzo de 2021. https://ijoc.org/index.php/ijoc/article/view/477

Sellas, Tony y Sergi Solà. 2019. "Podium Podcast and the freedom of podcasting: Beyond the limits of radio programming and production constraints". Radio Journal: International Studies in Broadcast \& Audio Media 17 (1): 63-81. https://doi.org/10.1386/rjao.17.1.63_1

Spotify. 2021. "About Spotify”. Fecha de consulta: 30 de agosto de 2021. https://newsroom.spotify.com/company-info/

Steven, McClung y Kristine Johnson. 2010. "Examining the Motives of Podcast Users". Journal of Radio and Audio Media 17 (1): 82-95.

https://doi.org/10.1080/19376521003719391

Sullivan, John. 2019. "The Platforms of Podcasting: Past and Present". Social Media + Society, 5 .

https://doi.org/10.1177/2056305119880002

Wiencek, Florian. 2018. "Digital Mediation of Art and Culture. A Database Approach”. Tesis de doctorado, Universidad de Jacobs, Bremen.

Para citar este texto:

Rodríguez Reséndiz, Perla Olivia. 2022. "Aproximaciones al estudio del pódcast como documento sonoro de origen digital". Investigación Bibliotecológica: archivonomía, bibliotecología e información 36 (90): 151-164.

http://dx.doi.org/10.22201/iibi.24488321xe.2022.90.58512 